moral judgements based only on 'medical risks.' Instead, capacity evaluators play the vital role of helping treaters recognise the true source of their moral distress. In those cases, referral to broader decision-making bodies such as ethics committees or the courts is appropriate.

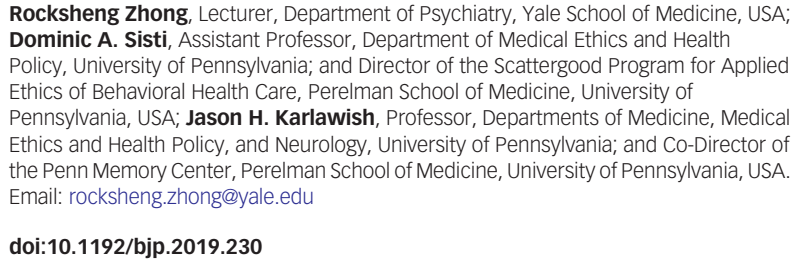

doi:10.1192/bjp.2019.230

\section{A contextual approach to routinely elicit a trauma-oriented history}

Thanks to Dr Ingrassia for her recent editorial on the Independent Inquiry into Child Sexual Abuse in the UK with an emphasis on the need for the sensitive and well-informed clinician to proactively and routinely ask about sexual abuse. ${ }^{1}$

In our study of child sexual abuse (CSA) history among psychiatric consultations in a general hospital emergency room, we found that $38 \%$ of individuals (adults and minors) referred for psychiatric consultation over a 2-year period described having experienced sexual abuse during their childhood. ${ }^{2}$

We used a semi-structured questionnaire with language that was appropriate to age and cultural background in order to routinely enquire whether the patient had experienced physical, emotional or sexual abuse during their childhood in accordance with a widely accepted definition of sexual abuse. ${ }^{3}$ This approach is consistent with the research that multiple forms of adverse childhood experiences may coexist. ${ }^{4}$ We believe that a contextual approach like this is more likely to promote a discussion of the person's trauma narrative. Using this paradigm, with appropriate training, it is hoped that medical and paramedical clinicians will be able to sensitively and routinely take a comprehensive trauma-oriented history in every patient. In this way, the patient's presenting problem may be understood and treated with an understanding of 'what has happened to this person'.

It is worth mentioning that adverse childhood experiences including sexual abuse is not only associated with an increase in lifetime prevalence of mental illness but also of physical illness. There is evidence linking early-life stress to reduced telomere length in a study of physically and psychiatrically healthy adults with or without a reported history of childhood trauma. These early experiences may affect adult health in two ways: either by cumulative damage over time or by the biological embedding of adversities during sensitive developmental periods. ${ }^{4}$ Mediating factors between CSA and physical illness include neuroendocrine dysfunction, metabolic syndrome and chronic inflammation. ${ }^{5}$

To the best of our knowledge our study is the first to investigate CSA history during hospital emergency room psychiatric consultations. It is hoped that there will be an increased awareness of CSA during psychiatric consultations in a general hospital setting.

Interventions for past CSA should include the nature of earlylife trauma and its effects on psychobehavioural factors. When healthcare providers counsel victims of childhood abuse, they should consider the long-term psychological and physical wellbeing necessary to counter adverse responses to abuse such as disordered eating, lack of exercise, sleeping problems and depressive symptoms. They should also promote healthier ways to cope with trauma. Such psychological interventions would have the potential to prevent or reduce physical health problems in later life. ${ }^{4}$
1 Ingrassia A. The Independent Inquiry into Child Sexual Abuse in the UK: reflecting on the mental health needs of victims and survivors. Br J Psychiatry 2018; 213: 571-3.

2 Jaworowski S, Golmard JL, Morag Engelberg M, Prijs S, Lital Twizer L, Gropp C, et al. Case-control retrospective study of child sexual abuse history among psychiatric consultations in a general hospital emergency room. IMAJ 2019; 21: 77-81.

3 Finkelhor D, Hotaling GT. Sexual abuse in the National Incidence Study of Child Abuse and Neglect: an appraisal. Child Abuse Neg/ 1984; 8: 23-32.

4 Shonkoff JP, Boyce WT, McEwen BS. Neuroscience, molecular biology, and the childhood roots of health. Disparities building a new framework for health promotion and disease prevention. JAMA 2009; 301: 2252-9.

5 Anda RF, Felitti VJ, Bremner JD, Walker JD, Whitfield C, Perry BD, et al. The enduring effects of abuse and related adverse experiences in childhood: a convergence of evidence from neurobiology and epidemiology. Eur Arch Psychiatry Clin Neurosci 2006; 256: 174-86.

Sol Jaworowski, Shaare Zedek Medical Centre, Israel; Cornelius Gropp, Psychiatrist, Shaare Zedek Medical Centre, Israel. Email: solj@szmc.org.il

doi:10.1192/bjp.2019.231

\section{Conclusions in Gryglewski et al may not be warranted}

A number of issues not addressed in Gryglewski et al require comment and clarification. ${ }^{1}$ First, the authors show that a significant increase in volumes in amygdala nuclei, hippocampus, putamen and cortical thickness occurred following a course of electroconvulsive therapy (ECT) in 12 patients. However, it is not stated whether these patients' brain structures average size at baseline is significantly different to what we would expect to find in a healthy cohort, or what percentage of the sample fall below the norm. If this is not clarified, we need to understand why brain structure sizes that may fall within a normal distribution would require enlarging.

Second, patients had two scans before ECT and the authors present the average of the two scans as baseline measures. The authors omit to say how different the measurements were between the two pre-ECT scans, which would inform the reader as to the accuracy of each magnetic resonance imaging reading. This is important since the same procedure was not employed at termination of treatment.

Third, the authors attribute the increase in volume to a process of neurogenesis, which they consider a positive outcome. However, they do not seem to take into account the possibility that the neurogenesis may not be benign but be the result of the electrical insult inflicted on the brain, and that the proliferation and morphology of the newly created neurons may not be normal. Neurogenesis has also been observed to occur in similar areas of the brain following intake of lithium and other mood stabilisers, but it was found that the number and morphology of the cells were abnormal, with increasing growth of cone formation, leading to the spreading of the neuron and a shorter neuronal axon'. ${ }^{2}$ If such cellular proliferation in the areas connected with memory is a positive outcome, rather than a pathological reaction to a brain insult, then widespread memory and cognitive impairment found in a large percentage of patients who have had $\mathrm{ECT}^{3}$ needs explaining.

Fourth, and related to the last point, there is no data presented on the incidence of adverse effects following ECT (disorientation, confusion, memory loss, concentration, impairment in abstract reasoning, overall level of cognitive functioning, docility, lethargy and apathy), which may impact on the ability to perform a post-treatment test.

Finally, the authors bemoan the difficulty with recruiting 'suitable patients' and ended up with a very small sample. In an era of antidepressant-induced treatment-resistant depression, ${ }^{4,5}$ I suspect 
that a fairly large number of patients in the University Clinic of Vienna would have met inclusion criteria. It is possible that other patients-related factors may have been involved in accounting for the very low sample size. In this respect, a wide gap between mainstream psychiatrists' views and patients' views regarding the usefulness of ECT has been revealed in a systematic review. ${ }^{6}$

1 Gryglewski G, Baldinger-Melich P, Seiger R, Godbersen GM, Michenthaler P, Klöbl M, et al. Structural changes in amygdala nuclei, hippocampal subfields and cortical thickness following electroconvulsive therapy in treatmentresistant depression: Iongitudinal analysis. Br J Psychiatry 2019; 214: 159-67.

2 Lagace DC, Eisch AJ. Mood-stabilizing drugs: are their neuroprotective aspects clinically relevant? Psychiatr Clin N Am 2005; 28: 399-414.

3 Sackeim HA, Prudic J, Fuller R, Keilp J, Lavori PW, Olfson M. The cognitive effects of electroconvulsive therapy in community settings. Neuropsychopharmacology 2007; 32: 244-54.

4 Fava GA. Can long-term treatment with antidepressant drugs worsen the course of depression? J Clin Psychiatry 2003; 64: 123-33.

5 Fava GA, Offidani E. The mechanisms of tolerance in antidepressant action. Prog Neuropsychopharmacol Biol Psychiatry 2011; 35: 1593-602.

6 Rose D, Fleischmann P, Wykes T, Leese M, Bindman J. Patients' perspectives on electroconvulsive therapy: systematic review. BMJ 2003; 326: 1363.

Marco Chiesa, Consultant Psychiatrist and Visiting Professor, University College London, UK. Email: m.chiesa@ucl.ac.uk

doi:10.1192/bjp.2019.232

\section{Response letter to the article by Killackey et al (2019) 'Individual placement and support for vocational recovery in first-episode psychosis: randomised controlled trial'}

It was with interest we read the results of the recent paper by Killackey et al evaluating the efficacy of individual placement and support (IPS) in first-episode psychosis (FEP) measured towards vocational outcomes and were excited to see positive results at earlier outcome points. ${ }^{1}$ The authors kindly offer multiple explanations of a loss of significance of such interventions at later end points, which we would like to contrast and expand upon.

The authors concluded that the research demonstrated a case for IPS in helping those with FEP return to work, despite this not being maintained at later time points. However, this may be optimistic given the key demographics examined could be potentially an overestimation.

First, the method of selection and randomisation denotes generous selection criteria but fails to fully account for an unequal distribution of affective presentations between group strata and self-selection of willing participants, suggesting a lower baseline functional impairment.

The evidence provided suggests short-term efficacy of IPS in motivated individuals of native language, with less severe symptoms, within a 'schizonormative' group and within a well-funded system. This is in keeping with previous research, but provides a relatively narrow margin for intervention, which does not clearly explain how and why it would be effective in those failing to make vocational recovery during usual treatment.
We would consider that those failing in the usual treatment arm may lie outside of the actionable group demonstrated, attributable to the coexistence of affective subtypes, more severe disease (omitted from the study), reduced language efficacy or other factors. Although some of this has evened out at later end-points, this may be because of other factors (remission of anergia, efficacy of pharmacological intervention). We would please request the authors address the above assertion.

Although the study does demonstrate some efficacy with those with a mood-affective component, as well as comorbid anxiety and post-traumatic stress disorder, unless further information is made available, it does not extrapolate the relevance of these factors into short- or long-term outcomes in a comprehensive way. We note that the control group included more affective presentations, which we hope have been accounted for.

The authors also comment that as a result of previous work, those operating in clinics may have been previously upskilled in IPS, which may also explain the high vocational rates ascribed to the treatment as usual arm and could have nullified significance at later end points. We would proffer an additional explanation, where some observer biases inherent in those clinics sharing both intervention and test cases would inspire more supportive treatment.

Finally, the measure of a successful outcome used may overestimate the success of the project, and the attenuation of employment in the intervention arm may further explain the loss of significance at later end points. We posit this could potentially empower the government into seeking more zero-hour contracts in the UK as evidence of employment. However, far from being a negative, it does suggest that IPS, and work around it, does favour positive outcomes and this has been reflected in long-term follow-up in a similar study in Switzerland ${ }^{2}$ and shown to improve outcomes. This suggests moreover that a team approach to support, regardless of vocational expert inclusion, may be the really successful intervention.

It may be that these programmes would present a viable intervention if tailored to adjust for affective symptoms, be maintained beyond 6 months, be 1:1, ${ }^{3}$ account for heterogeneity in comorbid substance use, account for patient aspirations, allow for differences between Australia and UK service structures and funding prospects, and include a team-based approach to service delivery.

1 Killackey E, Allott K, Jackson HJ, Scutella R, Tseng YP, Borland J, et al. Individual placement and support for vocational recovery in first-episode psychosis: randomised controlled trial. Br J Psychiatry 2019; 214: 76-82.

2 Hoffmann H, Jäckel D, Glauser S, Mueser KT, Kupper Z. Long-term effectiveness of supported employment: 5-year follow-up of a randomized controlled trial. Am J Psychiatry 2014; 171: 1183-90.

3 Holt RIG, Gossage-Worrall R, Hind D, Bradburn MJ, McCrone P, Morris T. Structured lifestyle education for people with schizophrenia, schizoaffective disorder and first-episode psychosis (STEPWISE): randomised controlled trial. Br J Psychiatry 2019; 214: 63-73.

B. M. Janaway, Core Psychiatric Trainee, National Hospital of Neurology and

Neurosurgery, UCLH, UK; Mukesh Kripalani, Consultant Psychiatrist, ADHD centre and PPCS, UK. Email: benjamin.janaway@nhs.net

doi:10.1192/bjp.2020.31 